\title{
A JUDICIALIZAÇÃO DO ACESSO AOS MEDICAMENTOS EM SANTA CATARINA: UM DESAFIO PARAA GESTÃO DO SISTEMA DE SAÚDE
}

The judicialization of access to medicines in Santa Catarina State: a challenge for the management of the health system

\author{
Alexandra Boing* \\ Neusa Sens Bloemer ${ }^{* *}$ \\ Claudia Roesler*** \\ Simone Fernandes ${ }^{* * * *}$
}

\begin{abstract}
RESUMO
O objetivo deste estudo foi analisar os mandados judiciais para fornecimento de medicamentos impetrados contra o Estado de Santa Catarina entre 2000 e 2006. As informações foram levantadas junto ao Programa de Medicamentos Judiciais (Mejud) da Secretaria Estadual de Saúde. Foi calculada a distribuição dos solicitantes por sexo, descritas as séries históricas do total de ações, o volume de recursos gastos pela Secretaria no período de 2000 a 2006 e se os medicamentos e/ou correlatos solicitados estavam padronizados no SUS. Também se calcularam as taxas de ações judiciais dos municípios por habitante a fim de testar a sua associação com indicadores socioeconômicos. No ano 2000 houve apenas uma ação deferida; em 2006 foram 1.661; de sete medicamentos solicitados em 2000, o total saltou para 3.542 em 2006. Destes, $29 \%$ eram padronizados pelo SUS. Os medicamentos mais solicitados destinavam-se ao tratamento do sistema nervoso e os que acarretaram maior custo foram os antineoplásicos. Municípios com melhores condições
\end{abstract}

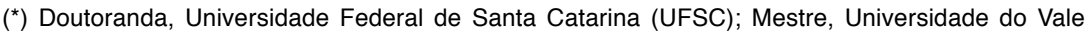
do Itajaí (Univali); Mestre, Universidade Federal de Santa Catarina (UFSC). Itajaí/SC - Brasil. E-mail: acboing@gmail.com.

$\left.{ }^{* *}\right)$ Doutora, Universidade do Vale do Itajaí (Univali). Itajaí/SC - Brasil.

${ }^{* * *}$ Doutora, Universidade do Vale do Itajaí (Univali). Itajaí/SC - Brasil.

$\left(^{\star \star \star \star}\right)$ Farmacêutica, Universidade Católica de Santos. Santos/SP - Brasil

Artigo recebido em: 24/05/2012. Revisado em: 15/08/2012. Aprovado em: 20/08/2012 
socioeconômicas apresentaram maior número de ações deferidas por habitante. Verificou-se necessidade de melhor articulação entre Executivo e Judiciário garantindo efetivação dos direitos constitucionais e uso racional dos medicamentos.

Palavras-chave: Assistência Farmacêutica; Poder Judiciário; Políticas Públicas de Saúde.

\section{ABSTRACT}

This study aims at analyzing the court orders granted that requested medication and were filed against the State of Santa Catarina from 2000 to 2006. The information was gathered from the Mejud, program of the Santa Catarina's State Health Secretary. The petitioners' gender distribution was calculated, a description was prepared of the historical series of the total lawsuits and of the volume of resources spent by the Secretary from 2000 to 2006, as well as whether the medication and supplies requested were standardized in SUS. The rate of lawsuits in Santa Catarina's cities was also calculated per capita in order to test their association with socioeconomic indexes through the Spearman correlation coefficient. In 2000 there was only one court order granted; in 2006 there were 1,661 ; in 2000 seven medicines were requested, and in 2006 there were 3,542 requests. The most highly sought medication was for the nervous system and the most expensive was antineoplastic medication. $29 \%$ of the medication was verified to be standardized by SUS. Cities with better socioeconomic conditions displayed a greater number of lawsuits granted per inhabitant.

Keywords: Health Public Policy; Judicial Power; Pharmaceutical Care.

\section{Introdução}

As políticas públicas de saúde no Brasil passaram por profundas alterações a partir da década de 1980, particularmente após a VIII Conferência Nacional de Saúde, um marco na estruturação do setor saúde no país por fortalecer as discussões sobre a responsabilidade do Estado quanto à saúde da população e por balizar a Constituição de 1988.

No tocante à assistência farmacêutica, têm havido muitas reformulações desde então. No período de análise deste trabalho a assistência farmacêutica esteve organizada em programas que, em certa medida, acompanhavam a organização da atenção à saúde em seus diferentes níveis de complexidade e/ou ações programáticas. A desarticulação entre os programas, as lacunas relacionadas aos elencos de medicamentos e os problemas estruturais nos serviços, entretanto, não possibilitaram o acesso universal aos medicamentos. Perdura uma situação de iniquidade em que os gastos privados familiares com medicamentos têm 
maior impacto para as camadas mais pobres da população. ${ }^{(1),(2)}$ Por outro lado, o sistema público tem sido a única alternativa para viabilizar o acesso a certos medicamentos, mesmo para camadas cobertas por planos de saúde privados. ${ }^{(3)}$

Nas situações não contempladas pelos programas governamentais, o fornecimento de medicamentos pelo Estado tem sido feito por meio do poder Judiciário. No cenário judicial constatam-se vários debates, sejam eles teóricos, sejam de ordem prática, em relação ao acesso ao medicamento pelo paciente. ${ }^{(4)}$ Por um lado, é citado o dever do Estado em garantir saúde aos cidadãos e a primazia da vida sobre qualquer outra perspectiva. Noutra vertente, citam-se os escassos investimentos públicos em saúde em contraposição à crescente demanda por medicamentos e a inclusão de novas tecnologias, em parte estimulada pela vasta gama de produtos farmacêuticos disponíveis no mercado e apologia ao uso indiscriminado de medicamentos na mídia leiga. ${ }^{(5)} \mathrm{O}$ atendimento a essas ações individuais pode, inclusive, colocar em xeque o sistema de saúde e o planejamento das ações coletivas. ${ }^{(6),(7),(8)}$

Diante do desafio que se antepõe às políticas públicas no setor saúde em geral, e à Assistência Farmacêutica em particular, de garantir saúde à população equilibrando-se os preceitos constitucionais do Sistema Único de Saúde (SUS) e do uso racional de medicamentos, é fundamental ampliarem-se os conhecimentos sobre o tema. O presente trabalho objetivou contribuir com essa perspectiva descrevendo características quantitativas referentes às ações judiciais deferidas pelo poder Judiciário para o acesso à assistência farmacêutica no Estado de Santa Catarina entre 2000 e 2006.

\section{Metodologia}

Os dados analisados se referem aos mandados judiciais deferidos pelo sistema jurídico e que foram impetrados contra o Estado de Santa Catarina para o

${ }^{1}$ SILVEIRA, F.G.; OSÓRIO, R.G.; PIOLA, S.F. Os gastos das famílias com saúde. Ciência \& Saúde Coletiva, São Paulo, v. 7, n. 4, p. 719-731, 2007.

2 ANDRADE, M.V.; NORONHA, K.V.M.S.; OLIVEIRA, T.B. Determinantes dos gastos das famílias com saúde no Brasil. Revista EconomiA, v. 7, n. 3, p. 485-508, set./dez. 2006.

${ }^{3}$ CONILL, E. M.; PIRES, D.; SISSON, M.C.; OLIVEIRA, M.C.D.; BOING, A.F.; FERTONANI, H.P. O mix público-privado na utilização de serviços de saúde: um estudo dos itinerários terapêuticos de beneficiários do segmento de saúde suplementar brasileiro. Ciência \& Saúde Coletiva, São Paulo, v. 13, n. 5, p. 1501-1510, 2008.

${ }^{4}$ AMARAL, G. Direito. Escassez \& Escolha. Rio de Janeiro: Renovar, 2001.

${ }^{5}$ NASCIMENTO, A.C. Ao persistirem os sintomas, o médico deverá ser consultado: isto é regulação? São Paulo: Sobravime, 2005.

${ }^{6}$ VIEIRA, F.S.; ZUCCHI, P. Distorções causadas pelas ações judiciais à política de medicamentos no Brasil. Revista de Saúde Pública, São Paulo, v. 41, n. 2, p. 214-222, 2007.

${ }^{7}$ MESSEDER, A.M., OSORIO, D.C.C.G.S.; LUIZA, V.L. Mandados judiciais como ferramenta para garantia do acesso a medicamentos no setor público: a experiência do Estado do Rio de Janeiro, Brasil. Cadernos de Saúde Pública, Rio de Janeiro, v. 21, n. 2, p. 525-34, 2005.

${ }^{8}$ MARQUES, S.B.; DALLARI, S.G. Garantia do direito social à assistência farmacêutica no Estado de São Paulo. Revista de Saúde Pública, São Paulo, v. 41, n. 1, p. 101-107, 2007. 
fornecimento de medicamentos e/ou correlatos entre 2000 e 2006 . Estes anos foram selecionados por compreender o período em que há disponibilidade de dados arquivados junto ao Programa de Medicamentos Judiciais (Mejud) na Diretoria de Assistência Farmacêutica (DIAF) da Secretaria do Estado da Saúde de Santa Catarina (SES/SC). O Mejud concentra as informações de todas as ações judiciais que condenaram o Estado a viabilizar medicamentos para o impetrante. Os dados analisados no presente estudo referem-se ao ano de início do processo (2000 a 2006), aos medicamentos e/ou insumos solicitados (princípio ativo/nome comercial e quantidade), aos gastos da secretaria com cada medicamento e ao sexo e ao município de residência do solicitante.

Para a realização dos procedimentos analíticos foi construído um banco de dados no programa estatístico Stata 9 (Stata Corp., College Station, Estados Unidos). A partir dele foi calculada a distribuição dos solicitantes por sexo e foram descritas as séries históricas do total de ações e do volume de recursos gastos pela SES/SC no atendimento das ações impetradas contra o Estado catarinense entre 2000 e 2006 solicitando medicamentos e/ou correlatos. Em seguida, verificou-se se os produtos solicitados estavam padronizados em algum programa governamental estadual e federal no período analisado. Também foi analisado em quais classes anatômicas terapêuticas houve maior demanda por medicamentos e que acarretaram maiores custos ao poder público. Para tal, os medicamentos foram agrupados em classes anatômico-terapêuticas, de acordo com a metodologia Anatomical Therapeutic Chemical (ATC), recomendada pela Organização Mundial da Saúde (OMS) e utilizada como padrão internacional. ${ }^{(9)}$

Por fim, calculou-se a taxa de ações judiciais por 100 mil habitantes para cada um dos 293 municípios catarinenses a fim de testar a sua associação com os indicadores socioeconômicos das localidades. Em tal análise calculou-se o coeficiente de correlação de Spearman e procedeu-se à distribuição espacial das taxas de ações judiciais de acordo com os quartis de sua distribuição e segundo a malha municipal disponibilizada pelo Instituto Brasileiro de Geografia e Estatística (IBGE). Esse procedimento foi conduzido através do programa TabWin 3.5. Os indicadores socioeconômicos são oriundos do Atlas do Desenvolvimento Humano no Brasil e referem-se ao ano $2000 .{ }^{(10)}$ No presente estudo foram utilizados os seguinte indicadores: índice de desenvolvimento humano municipal (IDH-M), coeficiente de Gini, renda per capita, intensidade de pobreza, mortalidade infantil, expectativa de vida, taxa bruta de frequência no ensino fundamental.

Os nomes dos sujeitos da pesquisa não foram identificados e o presente trabalho foi aprovado pela Diretoria de Assistência Farmacêutica do Estado de

\footnotetext{
9 WORLD HEALTH ORGANIZATION - WHO. The ATC classification structure and principles. Disponível em: <http://www.whocc.no/atcddd>. Acesso em: 02 mai. 2007.

10 PROGRAMA DAS NAÇÕES UNIDAS PARA O DESENVOLVIMENTO - PNUD. Atlas do Desenvolvimento Humano no Brasil. Brasília: PNUD, 2003.
} 
Santa Catarina e pelo Comitê de Ética da Universidade do Vale do Itajaí, sob protocolo número 397/2007.

\section{Resultados}

Observou-se que ao longo dos setes anos investigados houve um substancial aumento no número de ações deferidas, obrigando a Secretaria do Estado da Saúde de Santa Catarina a fornecer medicamentos, com consequente acréscimo também no total de medicamentos solicitados (Gráfico 1).

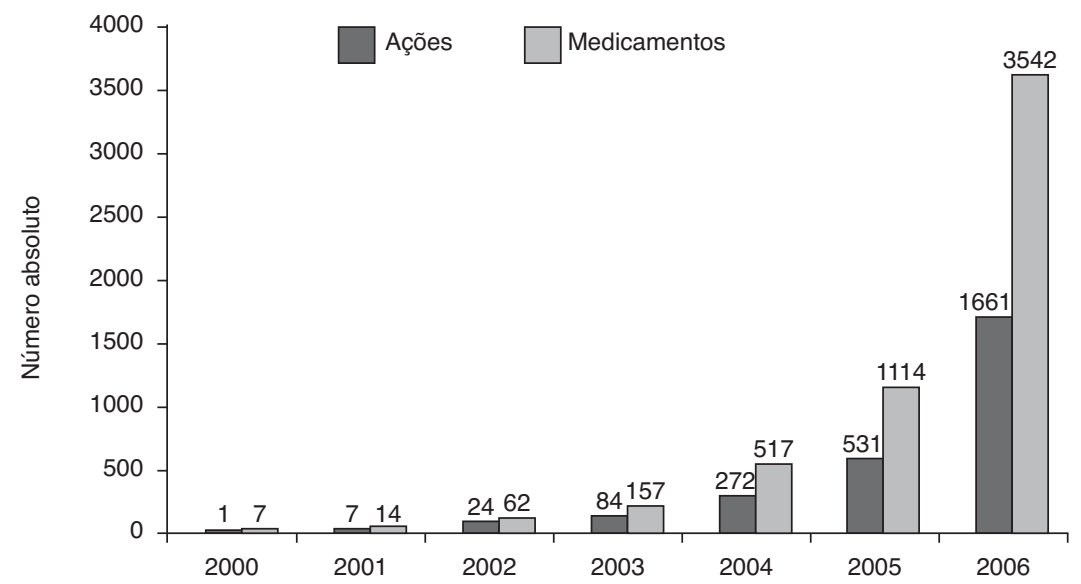

Gráfico 1. Série histórica do total de mandados judiciais deferidos requerendo a provisão de medicamentos pela Secretaria de Estado da Saúde de Santa Catarina e do total de medicamentos solicitados, 2000-2006.

No ano 2000 houve apenas uma ação deferida, enquanto em 2006 o total chegou a 1.661. Em relação ao número de medicamentos solicitados, o crescimento foi de apenas sete em 2000 para 3.542 em 2006. Cabe destacar o último biênio investigado. Em 2005, o número de ações e medicamentos foi de 531, triplicando em 2006. Ampliando um pouco mais o período, avaliando-se o último quadriênio, houve aumento de $1.878 \%$ no total de ações deferidas e $2.156 \%$ no total de medicamentos solicitados entre 2003 a 2006. Além disso, verificou-se que ao longo dos sete anos houve em média 2,2 medicamentos e/ou correlatos solicitados por processo e a quantidade máxima de produtos identificados em um único processo foi igual a 17, fazendo parte dos pleitos desde medicamentos até produtos como fraldas, luvas de látex, gaze, esparadrapo, tiras reativas de glicemia, entre outros. Também foi significante o acréscimo nos gastos da SES/SC para atender as demandas geradas pelos mandados deferidos. Entre os anos 2000 a 2006 os gastos relativos com esses itens foram majorados em $757.000 \%$. 
A classificação dos medicamentos segundo a metodologia ATC permitiu identificar que os mais solicitados foram aqueles referentes ao sistema nervoso, com 20,6\% das solicitações (Gráfico 2).

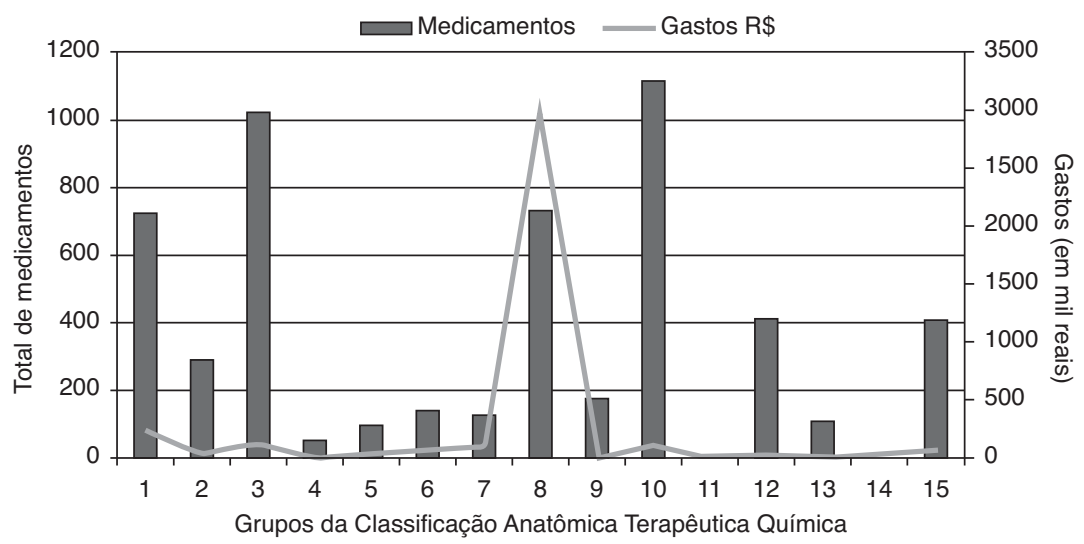

Gráfico 2. Total de medicamentos solicitados e de recursos gastos no atendimento aos mandados judiciais deferidos, requerendo a provisão de medicamentos pela Secretaria de Estado da Saúde de Santa Catarina, segundo a Classificação Anatômica Terapêutica Química, 2000-2006.

Em seguida apareceram os medicamentos do aparelho cardiovascular, com $19,0 \%$ das solicitações, e os medicamentos antineoplásicos e imunomoduladores (13,0\%). Apesar de ser apenas o terceiro quanto ao total de medicamentos solicitados, o grupo de agentes antineoplásicos e imunomoduladores compreendeu $77 \%$ do volume financeiro movimentado pela SES/SC no atendimento das ações entre 2000 e 2006.

A análise individual dos medicamentos demonstrou que os produtos mais solicitados durante os sete anos analisados foram o Adalimumab, seguido do Brometo Tiotrópio e da tira reativa para glicemia. Além disso, verificou-se que $29 \%$ dos medicamentos solicitados eram padronizados por algum programa governamental estadual ou federal.

Em relação aos custos, os três medicamentos que exigiram mais recursos à SES/SC foram Laronidase (totalizando gastos de 2000 a 2006 no valor de $\mathrm{R} \$$ $81.163,80)$, Temozolamida ( $\$$ \$ 34.764,30) e Infliximab $(R \$ 17.678,25)$. Dos 10 medicamentos mais solicitados, sete eram pertencentes à classe terapêutica dos antineoplásicos e imunomoduladores.

Em relação ao sexo do condutor da ação, verificou-se que 47,5\% eram homens e 52,5\% mulheres. Dos 293 municípios catarinenses, em 192 (65,5\%) houve ações impetradas contra o Estado entre 2000 e 2006 (Figura 1). 


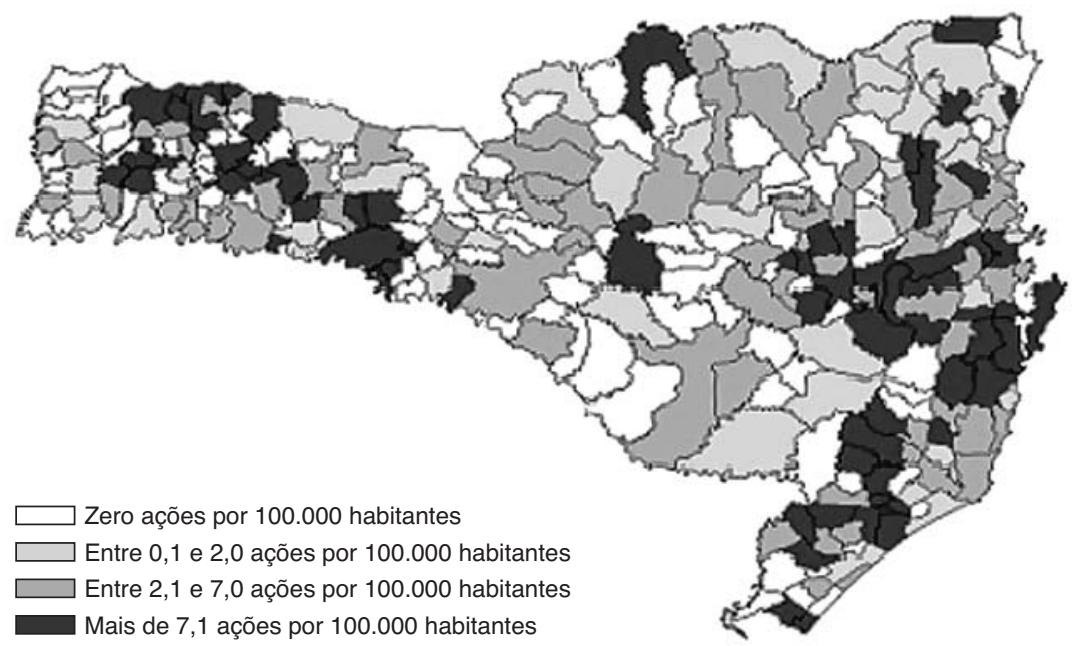

Figura 1. Distribuição espacial dos municípios catarinenses segundo os quartis da distribuição da relação entre as ações deferidas e o número de habitantes, 2000-2006.

Tabela 1. Matriz de correlação entre o número de ações per capita e indicadores socioeconômicos. Santa Catarina, 2000-2006.

\begin{tabular}{|c|c|c|c|c|c|c|c|c|}
\hline & APC & IDH & GINI & RPC & POB & MI & EV & FEF \\
\hline APC & 1 & $0,201^{\star *}$ & $-0,159^{* *}$ & $0,173^{\star *}$ & $-0,262^{\star *}$ & $-0,115^{\star \star}$ & $0,115^{\star *}$ & $0,200^{*}$ \\
\hline IDH & & 1 & $-0,217^{\star *}$ & $0,737^{\star *}$ & $-0,715^{\star \star}$ & $-0,618^{\star *}$ & $0,617^{\star *}$ & $0,317^{\star *}$ \\
\hline GINI & & & 1 & $-0,034$ & $0,512^{\star *}$ & $0,332^{\star *}$ & $-0,331$ & $-0,014$ \\
\hline RPC & & & & 1 & $-0,831^{\star *}$ & $-0,419^{\star \star}$ & $0,418^{\star *}$ & $0,237^{\star *}$ \\
\hline POB & & & & & 1 & $0,524^{\star *}$ & $-0,524^{\star *}$ & $-0,125^{*}$ \\
\hline MI & & & & & & 1 & $1,00^{* *}$ & $-0,033$ \\
\hline EV & & & & & & & 1 & $0,123^{*}$ \\
\hline FEF & & & & & & & & 1 \\
\hline
\end{tabular}

APC: ações per capita; IDH: Índice de Desenvolvimento Humano; GINI: coeficiente de Gini; RPC: renda per capita; POB: intensidade de pobreza; MI: taxa de mortalidade infantil; EV: expectativa de vida; FEF: taxa bruta de frequência no ensino fundamental ${ }^{*}$ Correlação estatisticamente significativa para $p<0,05$

${ }^{* *}$ Correlação estatisticamente significativa para $p<0,01$ 
Analisando a distribuição espacial dos municípios do estado onde houve ações movidas, verificamos que há concentração nas regiões próximas aos municípios da grande Florianópolis, Vale do Itajaí e Chapecó. Constatou-se, ainda, através do teste de correlação de Spearman, que os municípios com melhores indicadores socioeconômicos apresentaram maior número de ações deferidas por habitante (Tabela 1).

\section{Discussão}

O número de ações descritas no presente estudo são os incluídos no sistema Mejud da SES/SC. Entretanto, comparativamente aos valores anuais disponibilizados pelo Tribunal de Justiça de Santa Catarina, podem estar subestimados. Como consequência, tanto o número de ações deferidas quanto os valores gastos pelo poder público devem ser maiores do que os apresentados neste trabalho, indicando uma realidade ainda mais impactante. Estima-se que os valores gastos para cumprir as ações judiciais variaram ao longo do tempo, mas como exemplo do gasto catarinense pode-se citar que em 2006 foram gastos com assistência farmacêutica do Estado mais de $\mathrm{R} \$ 80$ milhões; deste valor, $\mathrm{R} \$ 21.483 .216,50$ foram referentes às decisões judiciais. ${ }^{(11)}$ É preciso considerar que o fenômeno da "judicialização da saúde" é relativamente novo no Estado, o que pode ser atestado, inclusive, pelo número praticamente insignificante de ações nos anos de 2000 e 2001. Por isso, ao longo do período investigado ocorreu o desenvolvimento e qualificação do sistema para a captação, armazenamento e gerenciamento das informações, o que representou um grande avanço na qualificação do serviço, com vistas ao seu uso como apoio para avaliação e planejamento de políticas públicas. De qualquer maneira, trabalhar com as informações oficiais dos sistemas de saúde e administração, explorando-os e tornando-os públicos, apesar de suas conhecidas limitações, é uma importante ferramenta no seu processo de qualificação e busca colaborar na discussão sobre a gestão dos dados.

De uma forma ou de outra, a direção é clara, inequívoca e indica ascensão da demanda judicial por medicamentos, conforme tendência nacional. ${ }^{(12),(13)} \mathrm{Em}$ 2003 o valor despendido pela União para compras de medicamentos por ordem judicial foi de $R \$ 188$ mil e em 2007, apenas até outubro, o valor chegou a $R \$$ 26 milhões. ${ }^{(14)}$ Nos cálculos elaborados pelo Ministério, com o valor gasto no atendimento das ações durante o ano de 2007 seria possível realizar cerca de três milhões de consultas médicas e mais 74 mil partos normais. Possivelmente

\footnotetext{
${ }^{11}$ SANTA CATARINA. SECRETARIA DE ESTADO DA SAÚDE. Plano Estadual de Saúde 2012-2015: Estado de Santa Catarina. Florianópolis: Secretaria de Estado da Saúde Florianópolis, 2012.

12 VIEIRA, F.S.; ZUCCHI, P. op. cit.

${ }^{13}$ MESSEDER, A.M.; OSORIO, D.C.C.G.S.; LUIZA, V.L. op. cit.

${ }^{14}$ JUNGMANN, M. Ministério da Saúde classifica como epidêmico volume de ações judiciais contra $o$ SUS. Brasília: Agência Brasil, 2007.
} 
os aumentos na solicitação de medicamentos e/ou correlatos podem estar associados ao maior nível de informação e organização da população, através, por exemplo, de associações de portadores de condições patológicas específicas que prestam assessoria jurídica para as pessoas com determinados agravos. ${ }^{(15)}$ Também não se pode desconsiderar a criação de uma verdadeira "indústria" da judicialização da saúde, com o estabelecimento de escritórios de advogados e consultórios médicos especializados em conduzir ações contra o Estado, além de grupos de portadores de doença explicitamente apoiados por laboratórios farmacêuticos, cujos interesses não se restringem, obviamente, à conquista do direito social à saúde. $O$ extremo dessa situação pode ser exemplificada com a recente deflagração de uma verdadeira quadrilha formada por advogados, um médico, administradores de uma organização não governamental e três funcionários de laboratórios multinacionais, acusada de mover processos fraudulentos contra o Estado de São Paulo para a obtenção de medicamentos por meio da via judicial. ${ }^{(16)}$

Tal fenômeno também pode ser parcialmente atribuído ao envelhecimento da população e à maior prevalência de doenças que exigem tratamento contínuo e oneroso, impossível de ser suportado pela renda das pessoas. ${ }^{(17)}$

O valor gasto pelo ente público na compra de medicamentos para atender às decisões judiciais, associado a um engessado e limitado orçamento para investimento em saúde e à Lei de Responsabilidade Fiscal - que estabelece limite ao gasto público -, compromete os recursos disponibilizados para ações coletivas básicas. Estas, por sua vez, são importantes instrumentos de promoção de saúde e prevenção de doenças e com grande potencial de evitar morbidades que, num Estado mais avançado, representarão custos ainda maiores ao governo e/ou ao enfermo. Quanto ao orçamento público em saúde, vale ressaltar que o montante investido no Brasil é relativamente baixo. No ano de 2003 houve investimento governamental em saúde de US\$270 per capita, valor bastante inferior ao de outros países, como Argentina (US\$ 518), Chile (US\$345), Colômbia (US\$439) e França (US\$ 2.213). ${ }^{(18)}$

O fato é que tal aumento de gastos com medicamentos representa enorme desafio aos planejadores e gestores, pois essas demandas e pressões realizadas pela indústria farmacêutica cresceram com grande intensidade num período relativamente curto, tornando difícil a tarefa de gestão no SUS. Cabe, assim, aos

\footnotetext{
${ }^{15}$ VIANNA, L.W.; BURGOS, M.B. Entre princípios e regras: cinco estudos de caso de Ação Civil Pública. Dados, São Paulo, v. 48, n. 4, p. 777-843, 2005.

${ }^{16}$ NOVE são presos acusados de aplicar o "golpe do remédio". Folha de S. Paulo, São Paulo, 2 out. 2008. Cotidiano, p. 1.

${ }^{17}$ MOTA, D.M.; SILVA, C.G.M.; ORTUN, V.S.C.E. Uso racional de medicamentos: uma abordagem económica para a tomada de decisões. Ciência \& Saúde Coletiva, São Paulo, v. 13, supl. 0, p.589601, 2007.

${ }^{18}$ WORLD HEALTH ORGANIZATION - WHO. World Health Report 2006: working together for health. Geneva: WHO, 2006.
} 
diversos atores envolvidos nesse processo a realização de debates em conjunto com a sociedade sobre todas as variáveis envolvidas para que possam atuar de maneira planejada e articulada, sem prejuízo aos princípios e diretrizes do SUS.

Já em relação à análise individual dos medicamentos e/ou correlatos mais solicitados, a constatação de que o Adalimumab representa o mais solicitado no período surpreende, uma vez que esse produto foi registrado no Brasil apenas em abril de 2003, inicialmente para o tratamento da artrite reumatoide. Posteriormente, a Agência Nacional de Vigilância Sanitária (Anvisa) também aprovou o seu uso no tratamento de espondilite anquilosante, artrite psoriásica e doença de Crohn, além de psoríase. Desde outubro de 2006, esse medicamento passou a integrar o componente de medicamentos especializados, para o tratamento da artrite reumatoide, no Estado de Santa Catarina. De qualquer forma, o fato de esse produto ter-se tornado o mais demandado no período demonstra que sua incorporação nas prescrições médicas deu-se imediatamente após o seu registro, o que sugere sua ampla divulgação no meio médico.

O segundo medicamento mais demandado foi o Brometo de Tiotrópio. Esse produto, indicado para o tratamento de manutenção de pacientes com Doença Pulmonar Obstrutiva Crônica (DPOC), não consta da Relação Nacional de Medicamentos Essenciais (Rename), nem tampouco integra os componentes de financiamento da assistência farmacêutica. Até o ano de 2012 não consta a sua inclusão na lista de medicamentos do componente especializado do Estado de Santa Catarina nem nos protocolos clínicos e diretrizes terapêuticas do Ministério da Saúde, não estando o protocolo disponibilizado no primeiro semestre de 2012, pois se encontrava em consulta pública. Segundo a consulta pública $n^{\circ} 4$ de maio de 2012 do Protocolo Clínico e Diretrizes Terapêuticas - Doença Pulmonar Obstrutiva Crônica, nas revisões realizadas, o brometo de tiotrópio não apresentou superioridade clínica quando comparado ao formoterol ou ao salmeterol associado com ou sem corticosteroide, além de estudos demonstrarem aumento do risco de mortalidade entre os usuários do brometo de tiotrópio, fato esse que inviabiliza a inclusão desse medicamento, segundo o protocolo disponibilizado na consulta pública pelo Ministério da Saúde.

A tira reativa para glicemia, terceiro produto mais solicitado, é utilizada para teste de glicemia em pacientes diabéticos para controle da doença e não constava na lista do SUS na época do estudo. Esse correlato é importante para o acompanhamento domiciliar e consequente controle do diabetes. Mas é importante ressaltar que no final de 2006 o Ministério da Saúde criou a Lei n 11.347/06, , $^{(19)}$ a qual disponibiliza tanto o glicosímetro, que é o aparelho que mede o nível de glicose no sangue, quanto as tiras reativas para o teste, para ajudar os pacientes cadastrados no HiperDia quanto ao controle da doença no País. Essa distribuição, no entanto, ainda não ocorreu via estado catarinense, mas

\footnotetext{
${ }^{19}$ BRASIL. Lei $n^{\circ} 11.347$, de 27 de setembro de 2006. "Dispõe sobre o fornecimento de medicamentos e materiais necessários para aplicação e monitoramento do diabetes". Disponível em: <http://www. planalto.gov.br/ccivil_03/_Ato2004-2006/2006/Lei/L11347.htm>. Acesso em: 29 mar. 2012.
} 
recentemente muitos municípios de Santa Catarina disponibilizaram a tira reativa nas unidades básicas de saúde. Em relação à Laronidase e à Temozolamida, estes medicamentos não foram incluídos na lista de medicamentos especializados do estado catarinense. A Laronidase, utilizada para mucopolissacaridoses (MPS), não foi incluída nos protocolos clínicos ainda; tal fato pode ser justificado pela não existência de aumento de sobrevida e os estudos desenvolvidos não serem consistentes para tal inferência. ${ }^{(20)}$ Já a Temozolamida, relatório técnico do departamento de atenção especializada do Ministério da Saúde indica que este medicamento não demonstra eficácia comparativa melhor ou superior aos tratamentos já consagrados para tumores cerebrais.

A crescente solicitação dos medicamentos que estão inseridos em programas e na Rename pode demonstrar alguns possíveis problemas, como: (i) falta de medicamentos na rede básica; (ii) burocracia para se conseguir participar dos programas; (iii) não conhecimento por parte do prescritor dos programas governamentais existentes; e (iv) não enquadramento do indivíduo solicitante nos protocolos clínicos. Alguns desses pontos foram evidenciados em 2003 durante a Conferência Estadual de Medicamentos, onde foi apontada por parte dos usuários a limitação de medicamentos disponíveis no SUS, tanto pela quantidade como pela relação de produtos, além de existir grande burocracia e a necessidade de melhor divulgação das listas entre os profissionais de saúde. ${ }^{(21)}$

Em muitos casos, mesmo sendo de responsabilidade do município o fornecimento do medicamento, observou-se no presente estudo que muitos estão sendo fornecidos pelo Estado devido às decisões judiciais. Como exemplo cita-se o Paracetamol, um medicamento comumente utilizado na atenção básica como analgésico e que não deveria ser autorizado para fornecimento pelo Estado, e sim pelo município, pois, conforme definido pela Norma Operacional da Assistência à Saúde (NOAS/2002) e pelo Pacto de Gestão de 2006, (22),(23) esse medicamento é de responsabilidade desse ente. Tais confusões comprometem a gestão dos serviços de saúde, trazendo sérias implicações na concretização das políticas públicas, pois dificultam não só a provisão, mas também o planejamento dos recursos, gerando maiores dificuldades para a concretização de ações de saúde e fortalecimento do SUS.

${ }^{20}$ DINIZ, D.; MEDEIROS, M.; SCWARTZ, I.V. Consequências da Judicialização das políticas de saúde: custo de medicamentos para as mucopolissacaridoses. Cadernos de Saúde Pública, Rio de Janeiro, v. 28, n. 3, p. 479-489, 2012.

21 SANTA CATARINA. SECRETARIA DE ESTADO DA SAÚDE. Diretoria de Planejamento e Coordenação. Plano Estadual de Assistência Farmacêutica. Florianópolis: Secretaria de Estado da Saúde de Florianópolis, 2005.

22 BRASIL. MINISTÉRIO DA SAÚDE. Secretaria de Assistência à Saúde. Regionalização da Assistência à Saúde: aprofundando a descentralização com equidade no acesso. Norma Operacional da Assistência à Saúde: NOAS-SUS. Brasília-DF: Ministério da Saúde, 2001.

${ }^{23}$ BRASIL. MINISTÉRIO DA SAÚDE. Secretaria Executiva. Diretrizes Operacionais dos Pactos pela Vida: em defesa do SUS e de Gestão. Brasília: Ministério da Saúde, 2006. 
Situação semelhante ocorre com os pedidos referentes aos correlatos, como fraldas, luvas e esparadrapos, revelando que podem existir lacunas importantes na cobertura de insumos e correlatos imprescindíveis na atenção à saúde. Alguns insumos como esparadrapos e luvas são utilizados para troca de curativos, sendo este procedimento oferecido nas unidades de saúde ou realizado no próprio domicílio da pessoa através das equipes de saúde. Já as fraldas, sobretudo as geriátricas, utilizadas em pacientes são produtos individuais necessários para uma melhor qualidade de vida para as pessoas que possuem a necessidade de utilizá-las.

Sob a lógica de mercado e visando aumento do faturamento, a indústria farmacêutica lança a cada ano uma grande quantidade de produtos farmacêuticos e difunde a imagem de capacidade e inovação tecnológica. Em 2006 existiam disponibilizadas no mercado cerca de 15 mil apresentações farmacêuticas, e entre 2002 e 2006 surgiram mais de seis mil produtos novos no mercado. Muitas dessas supostas inovações, no entanto, não possuem diferencial em relação a outros medicamentos já estabelecidos. ${ }^{(24),(25)}$ Entre 1998 e 2002, foram aprovados 415 novos medicamentos pela Food and Drug Administration (FDA), mas somente $14 \%$ eram realmente inovadores, ou seja, apresentaram avanços significativos. ${ }^{(26)}$ Assim, através da propaganda ou marketing disfarçado de "informação educativa", as indústrias farmacêuticas conseguem influenciar e condicionar comportamentos de grande parte dos indivíduos e profissionais de saúde, apesar de muitas vezes os medicamentos inovadores não possuírem melhorias significantes em comparação com os já existentes no mercado. ${ }^{(27)}$

Constatou-se no presente estudo que houve associação positiva em nível municipal entre melhores condições socioeconômicas e mandados deferidos por habitante. Achado similar foi descrito por Vieira e Zucchi(28) no município de São Paulo em 2006. As autoras observaram que $63 \%$ das pessoas que impetraram ações contra o município de São Paulo residiam em área de menor grau de exclusão social. A partir dos dados analisados no Estado catarinense no período de 2000 a 2006 abrem-se algumas possibilidades, dentre as quais: (i) os municípios com melhores indicadores socioeconômicos, apesar disso, apresentam baixa provisão de medicamentos e/ou elevadas prevalências de patologias, fazendo com que sua população necessite recorrer à justiça para garantir seu tratamento; ou (ii) nos municípios com melhores condições socioeconômicas há também maior facilidade de acesso por parte da população aos mecanismos judiciais devido à

\footnotetext{
${ }^{24}$ MARIN, L.; LUZIA, V.L.; OSORIO, D.C.C.G.S.; SANTOS, S.M. (Orgs). Assistência Farmacêutica para gerentes municipais. Brasília: OPAS, 2003.

${ }^{25}$ LA REVUE PRESCRIRE. Política industrial ou saúde pública: o abismo aumenta. Tradução de Marcelo Rouanet e José Ruben de Alcântara Bonfim. Boletim Sobravime, n. 40/41, p. 10-19, 20012004.

${ }^{26}$ ANGELL, M. A verdade sobre os laboratórios farmacêuticos. Rio de Janeiro; São Paulo: Record, 2007.

${ }^{27}$ TEMPORÃO, J.G. A propaganda de medicamentos e o mito da saúde. Rio de Janeiro: Graal, 1986.

${ }^{28}$ VIEIRA, F.S.; ZUCCHI, P. op.cit.
} 
estrutura judiciária e/ou ao grau de instrução e organização das pessoas. A primeira assertiva é pouco provável, uma vez que a literatura é farta em referências indicando que municípios com melhores indicadores socioeconômicos também apresentam melhores indicadores populacionais de saúde.(29),(30),(31) Já a segunda assertiva pode indicar que os municípios com melhores condições socioeconômicas conseguem prover maior acesso à rede de serviços assistenciais, bem como à estrutura judiciária, devido à sua melhor organização e/ou maior divulgação de informações e consequentemente maior e melhor nível de organização social. Estudo conduzido em Minas Gerais em 2007 indicou que os indivíduos com meIhores condições socioeconômicas podem arcar com as despesas processuais e apresentar maior conhecimento sobre seus direitos e, por isso, proporcionalmente são os indivíduos que mais impetram ações solicitando medicamentos. ${ }^{(32)}$

De qualquer maneira, verificou-se a existência, no nível ecológico, de diferenças estatisticamente significantes quanto ao número relativo de ações deferidas, podendo indicar iniquidades que poderão repercutir no aumento das iniquidades nos perfis de saúde e socioeconômicos da população, já que maior proporção dos processos é impetrada por pacientes com menor grau de exclusão, ${ }^{\left({ }^{(3)}\right)}$ podendo assim agravar ainda mais a desigualdade no acesso aos medicamentos, aumentando os gastos públicos e diminuindo os investimentos coletivos. Outra questão relacionada ao perfil de saúde e socioeconômico da população é que sem acesso ao medicamento ela acaba por comprometer consideravelmente parte de sua renda com medicamentos, ${ }^{(34)}$ e muitas vezes sem este acesso acaba por ter uma piora no seu estado de saúde.(35)

Vale ressaltar que o delineamento do presente estudo não permitiu identificar se são pessoas mais privilegiadas que estão recorrendo à esfera judicial para obter medicamentos. Investigações que procedam à análise no nível individual são desejáveis para clarear essa questão.

${ }^{29}$ BALDANI, M.H.; NARVAI, P.C.; ANTUNES, J.L.F. Cárie dentária e condições socioeconômicas no Estado do Paraná, Brasil, 1996. Cadernos de Saúde Pública, Rio de Janeiro, v. 18, n. 3, p. 755763, 2002.

${ }^{30}$ ICHITANI, L.H.; FRANCO, G.G.; PERPÉTUO, I.H.A.; FRANÇA E. Desigualdade social e mortalidade precoce por doenças cardiovasculares no Brasil. Revista de Saúde Pública, São Paulo, v. 40, n. 4, p. 684-691, 2006.

${ }^{31}$ BEZERRA, F.J.G.; PONTES, L.R.F.S.K.; MINÁ, D.L.; BARRETO, M.L. Mortalidade infantil e condições sociodemográficas no Ceará, em 1991 e 2000. Revista de Saúde Pública, São Paulo, v. 41, n. 6, p.1023-1031, 2007.

${ }^{32}$ MACHADO, Marina Amaral de Ávila et al. Judicialização do acesso a medicamentos no Estado de Minas Gerais, Brasil. Revista de Saúde Pública, São Paulo, v. 45, n. 3, p. 590-598, 2011.

${ }^{33}$ CHIEFFI A.L.; BARATA, R.B. Judicialização da política pública de assistência farmacêutica e equidade. Cadernos de Saúde Pública, Rio de Janeiro, v. 25, n. 8, p. 1839-1849, 2009.

${ }^{34}$ BOING, A.C.; BERTOLDI, A.D.; PERES, K.G. Desigualdades socioeconômicas nos gastos e comprometimento de renda com medicamentos no Sul do Brasil. Revista de Saúde Pública, São Paulo, v. 45, n. 5, p. 897-905, 2011.

${ }_{35}$ INSTITUTO BRASILEIRO DE GEOGRAFIA E ESTATÍSTICA - IBGE. Pesquisa de Orçamentos Familiares 2002-2003. Rio de Janeiro: IBGE; 2004. 
Os dados apresentados neste trabalho evidenciam o crescente número de ações impetradas contra o Estado, aumento dos gastos, grande número de medicamentos padronizados concedidos por via judicial e maior número de ações impetradas por munícipes de cidades com melhores condições socioeconômicas. Esses fatos demonstram a necessidade de maior e melhor articulação entre Executivo e Judiciário devido à limitação de recursos, aumento de demanda e sobreposição de necessidades individuais versus as necessidades coletivas. Mesmo com os avanços conquistados nos últimos anos, como a ampliação dos protocolos clínicos, enquanto em 2002 o Ministério da Saúde apresentava sete protocolos, em 2012 são mais de 70 protocolos clínicos existentes. O conselho nacional de justiça instituiu um fórum nacional de monitoramento e solução das demandas relativas à assistência a saúde, por ser considerado o tema da judicialização desafiador para o poder Judiciário, gerando aumento vertiginoso das ações judiciais no país. A criação da Comissão Nacional de Incorporação de Tecnologia no SUS (Conitec) também representa um avanço importante à medida que é responsável pela incorporação, exclusão ou alteração de novos medicamentos, produtos, procedimentos, bem como responsável pelas alterações nos protocolos clínicos de diretrizes terapêuticas. Já a responsabilidade do poder Executivo deve ser a elaboração e implementação dessas políticas de saúde para que o cidadão não fique desprovido de assistência, enquanto o poder Judiciário deverá, quando lhe couber, garantir o direito do cidadão através do controle da constitucionalidade, sem olvidar as políticas públicas já existentes. Essa melhor articulação entre os poderes tenciona para que os princípios da integralidade, universalidade e equidade não sejam quebrados e as políticas públicas sociais e econômicas sejam efetivas e tenham suas ações voltadas para o bem coletivo, garantindo assim a efetivação dos direitos garantidos na constituição e o uso racional dos medicamentos.

\section{Referências}

AMARAL, G. Direito, escassez \& escolha. Rio de Janeiro: Renovar, 2001.

ANDRADE, M.V.; NORONHA, K.V.M.S.; OLIVEIRA, T.B. Determinantes dos gastos das famílias com saúde no Brasil. Revista EconomiA, v. 7, n. 3, p. 485508, set./dez. 2006.

ANGELL, M. A verdade sobre os laboratórios farmacêuticos. Rio de Janeiro; São Paulo: Record, 2007.

BALDANI, M.H.; NARVAI, P.C.; ANTUNES, J.L.F. Cárie dentária e condições socioeconômicas no Estado do Paraná, Brasil, 1996. Cadernos de Saúde Pública, Rio de Janeiro, v. 18, n. 3, p. 755-763, 2002. 
BEZERRA, F.J.G.; PONTES, L.R.F.S.K.; MINÁ, D.L.; BARRETO, M.L. Mortalidade infantil e condições sociodemográficas no Ceará, em 1991 e 2000. Revista de Saúde Pública, São Paulo, v. 41, n. 6, p.1023-1031, 2007.

BOING, A.C.; BERTOLDI, A.D.; PERES, K.G. Desigualdades socioeconômicas nos gastos e comprometimento de renda com medicamentos no Sul do Brasil. Revista de Saúde Pública, São Paulo, v. 45, n. 5, p. 897-905, 2011.

BRASIL. MINISTÉRIO DA SAÚDE. Secretaria de Assistência à Saúde. Regionalização da Assistência à Saúde: aprofundando a descentralização com equidade no acesso. Norma Operacional da Assistência à Saúde: NOAS-SUS. Brasília-DF: Ministério da Saúde, 2001.

Secretaria Executiva. Diretrizes Operacionais dos Pactos pela Vida: em defesa do SUS e de Gestão. Brasília: Ministério da Saúde, 2006.

CHIEFFI, A.L.; BARATA, R.B. Judicialização da política pública de assistência farmacêutica e equidade. Cadernos de Saúde Pública, Rio de Janeiro, v. 25, n. 8, p. 1839-1849, 2009.

CONILL, E.M.; PIRES, D.; SISSON, M.C.; OLIVEIRA, M.C.; BOING, A.F.; FERTONANI, H.P. O mix público-privado na utilização de serviços de saúde: um estudo dos itinerários terapêuticos de beneficiários do segmento de saúde suplementar brasileiro. Ciência \& Saúde Coletiva, São Paulo, v. 13, n. 5, p. 1501-1510, 2008.

DINIZ, D.; MEDEIROS, M.; SCWARTZ, I.V. Consequências da Judicialização das políticas de saúde: custo de medicamentos para as mucopolissacaridoses. Cadernos de Saúde Pública, Rio de Janeiro, v. 28, n. 3, p. 479-489, 2012.

ICHITANI, L.H.; FRANCO, G.G.; PERPÉTUO, I.H.A.; FRANÇA, E. Desigualdade social e mortalidade precoce por doenças cardiovasculares no Brasil. Revista de Saúde Pública, São Paulo, v. 40, n. 4, p. 684-691, 2006.

INSTITUTO BRASILEIRO DE GEOGRAFIA E ESTATÍSTICA - IBGE. Pesquisa de Orçamentos Familiares 2002-2003. Rio de Janeiro: IBGE; 2004.

JUNGMANN, M. Ministério da Saúde classifica como epidêmico volume de ações judiciais contra o SUS. Brasília: Agência Brasil, 2007.

LAREVUE PRESCRIRE. Política industrial ou saúde pública: o abismo aumenta. Tradução de Marcelo Rouanet e José Ruben de Alcântara Bonfim. Boletim Sobravime, n. 40/41, p. 10-19, 2001-2004.

MACHADO, Marina Amaral de Ávila et al. Judicialização do acesso a medicamentos no Estado de Minas Gerais, Brasil. Revista de Saúde Pública, São Paulo, v. 45, n. 3, p. 590-598, 2011.

MARIN, L.; LUZIA, V.L.; OSORIO, D.C.C.G.S.; SANTOS, S.M. (Orgs). Assistência farmacêutica para gerentes municipais. Brasília: OPAS, 2003. 
MARQUES, S.B.; DALLARI, S.G. Garantia do direito social à assistência farmacêutica no Estado de São Paulo. Revista de Saúde Pública, São Paulo, v. 41, n. 1, p. 101-107, 2007.

MESSEDER, A.M.; OSORIO, D.C.C.G.S.; LUIZA, V.L. Mandados judiciais como ferramenta para garantia do acesso a medicamentos no setor público: a experiência do Estado do Rio de Janeiro, Brasil. Cadernos de Saúde Pública, Rio de Janeiro, v. 21, n. 2, p. 525-34, 2005.

MOTA, D.M.; SILVA, C.G.M.; ORTUN, V.S.C.E. Uso racional de medicamentos: uma abordagem económica para a tomada de decisões. Ciência \& Saúde Coletiva, São Paulo, v. 13, supl. 0, p. 589-601, 2007.

NASCIMENTO, A.C. Ao persistirem os sintomas, o médico deverá ser consultado: isto é regulação? São Paulo: Sobravime, 2005.

NOVE são presos acusados de aplicar o "golpe do remédio". Folha de S. Paulo, São Paulo, 02 out. 2008. Cotidiano, p. 1.

PROGRAMA DAS NAÇÕES UNIDAS PARA O DESENVOLVIMENTO - PNUD. Atlas do Desenvolvimento Humano no Brasil. Brasília: PNUD, 2003.

SANTA CATARINA. SECRETARIA DE ESTADO DA SAÚDE. Diretoria de Planejamento e Coordenação. Plano Estadual de Assistência Farmacêutica. Florianópolis: Secretaria de Estado da Saúde de Florianópolis, 2005.

Plano Estadual de Saúde 2012-2015: Estado de Santa Catarina. Florianópolis: Secretaria de Estado da Saúde Florianópolis, 2012.

SILVEIRA, F.G.; OSÓRIO, R.G.; PIOLA, S.F. Os gastos das famílias com saúde. Ciência \& Saúde Coletiva, São Paulo, v. 7, n. 4, p. 719-731, 2007.

TEMPORÃO J.G. A propaganda de medicamentos e o mito da saúde. Rio de Janeiro: Graal, 1986.

VIANNA, L.W.; BURGOS, M.B. Entre princípios e regras: cinco estudos de caso de Ação Civil Pública. Dados, São Paulo, v. 48, n. 4, p. 777-843, 2005.

VIEIRA, F.S.; ZUCCHI, P. Distorções causadas pelas ações judiciais à política de medicamentos no Brasil. Revista de Saúde Pública, São Paulo, v. 41, n. 2, p. 214-222, 2007.

WORLD HEALTH ORGANIZATION - WHO. The ATC classification structure and principles. Disponível em: <http://www.whocc.no/atcddd>. Acesso em: 02 mai. 2007. 2006.

World Health Report 2006: working together for health. Geneva: WHO, 